\title{
Research on High Speed Parallel Blind Equalization Algorithm
}

\author{
Hao LI, Zhi-gang WANG and Hou-jun WANG \\ School of Automation Engineering, University of Electronic Science and Technology of \\ China, Chengdu 611731, China \\ Email: haoli_uestc@163.com
}

Keywords: Parallel blind equalization algorithm, CMA, Coordinate transformation.

\begin{abstract}
The paper presents an efficient high speed parallel blind equalization algorithm suitable for multi-mode signals in wireless communication system. The parallel algorithm combines the idea of coordinate transformation on the basis of the classical CMA. It converts a multi-mode signals into a constant-mode signal, so that the system error can be more toward zero. We also give a detailed implementation structure of the parallel blind equalization algorithm. The simulation results show that the proposed parallel blind equalization algorithm has excellent performance for modulated multi-mode signals, and the mean square error (MSE) value of the proposed algorithm is smaller than the classic CMA about $10 \mathrm{~dB}$.
\end{abstract}

\section{Introduction}

As one of the key technologies in wireless communication system, equalization technology has been widely studied. In the actual communication system, the non-idealization of the channel and the digital filter's truncation will cause the inter symbol interference (ISI); the limited bandwidth of the channel and the multipath effect of the channel can also cause ISI. These factors can result in a sharp decline in performance and seriously affect the quality of communications. Therefore, it is indispensable to use equalization techniques to reduce or eliminate ISI, that is, to design a filter to eliminate non-ideal channel effects.

Usually, the equalizer is implemented in frequency domain and time domain. The frequency domain equalizer mainly satisfies the distortion-free transmission condition by correcting the system amplitude frequency characteristic and group delay. Its implementation is complex, so it is generally not used for wireless communication systems. From the time domain perspective, time domain equalizer makes the impulse response of the whole system satisfy the condition of no ISI. The traditional time domain equalizer is implemented in serial form and need to receive training sequences separately [1,2], but this reduces the utilization of the channel bandwidth. Since the influence of the general channel is unpredictable and time varying, so a blind equalization algorithm is first proposed in [3]. In the case of no training sequence, the blind equalization algorithm can dynamically track the channel changes and update the equalizer coefficients in time. This feature greatly improves the efficiency of the algorithm and more suitable for complex scenarios. Godard proposed a constant modulus blind equalization algorithm [4], which applies to all the constant envelope of the transmitted signal. Constant Modulus Algorithm (CMA), as a special case of Godard algorithm is shown $[5,6,7]$. It has become a classic blind equalization algorithm, due to the low computational complexity and easy to achieve and so on. But it only applies to signal with constant modulus values, such as 4QAM, 2PAM. When a higher communication rate is required, it is unavoidable to use a signal modulation format with 
high channel utilization, such as 16QAM. However, for such multi-mode signals, although it can converge with the classic CMA, but the error function will never become zero. And its convergence speed rate is slow and steady state error is large [8]. Simultaneously, with the development of high speed communications, the traditional serial equalization algorithm has been unable to meet the demands of high speed data transmission. Thus, an efficient parallel algorithm architecture for multi-mode signals is necessary.

In this paper, a new fully high speed parallel blind equalization algorithm is presented. In the error estimation module, we propose an improved CMA, which is based on the classical CMA and the idea of coordinate transformation. Compared to existing classical CMA, the improved CMA has a unique advantage for multi-mode signals. And it converts a multi-mode signals into a constant-mode signal. In addition, the whole parallel blind equalization algorithm structure is given in detail. It has perfect practical value for the application of parallel blind equalization algorithm.

The rest of this paper is organized as follows. Section II introduces the traditional serial equalization algorithm. Section III describes the parallel blind equalization algorithm and its parallel implementation structure. Section IV shows the results of simulation. Finally, conclusions are drawn in Section V.

\section{Serial Equalization Algorithm}

The essence of the equalizer is a compensation filter that compensates for ISI caused by the channel. The traditional serial equalizer structure is shown as in Fig.1. In Fig.1, the serial equalizer mainly includes the filter module, coefficient update module, error estimation module.

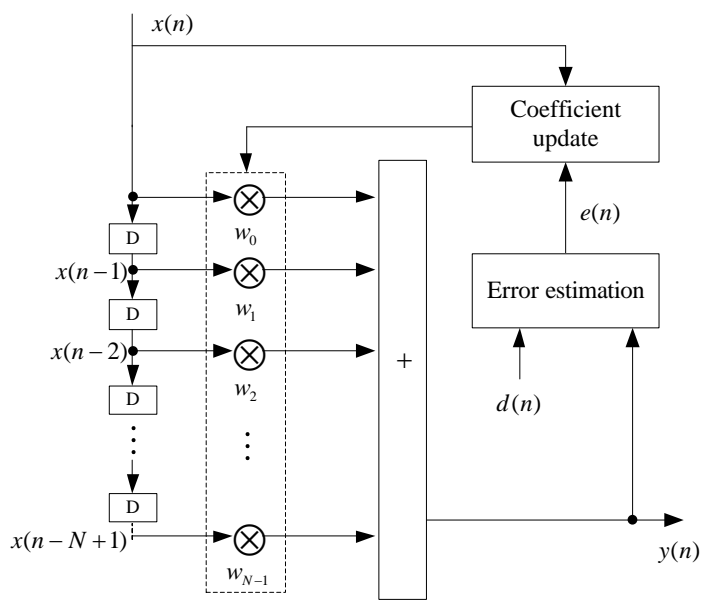

Figure 1. Serial equalizer structure

We assume that the time domain continuous signal is $x(t)$ at the receiving side. After the ADC sampling, the input signal of the equalizer is $x(n)$. In Fig.1, D represents the delay unit. $w_{i}(n),(0 \leq i \leq N-1)$ is the equalizer coefficient and $N$ is the equalization filter length. The expression of the equalizer output $y(n)$ is as follows

$$
y(n)=\sum_{i=0}^{N-1} x(n-i) w_{i}(n)
$$


In order to eliminate the ISI at the receiving side, it should select the appropriate equalizer coefficient $w_{i}(n)$.

In the traditional serial equalizer, the least mean square (LMS) algorithm is adopted [9]. The coefficient update module is a difference equation, the expression is as follows

$$
w_{i}(n+1)=w_{i}(n)+\mu e(n) x^{*}(n-i)
$$

where $\mu$ denotes the update step size, which controls the convergence characteristics of the equalization algorithm. The $e(n)$ denotes the error function. And the $X^{*}(n)$ represents the conjugate of the $X(n)$.

The error function $e(n)$ is defined as

$$
e(n)=d(n)-y(n)
$$

where $d(n)$ is the desired output value of the equalizer.

The purpose of LMS algorithm is to make the mean square error (MSE) $J$ tends to zero by adjusting the equalizer coefficient $w_{i}(n)$. Then $w_{i}(n)$ must satisfy the following condition.

$$
\left\{\begin{array}{l}
J=E\left[e^{2}(n)\right] \\
\frac{\partial J}{\partial w_{i}(n)}=0
\end{array}\right.
$$

Further simplified as follows

$$
\begin{aligned}
2 E\left[e(n) \frac{\partial e(n)}{\partial w_{i}(n)}\right] & =-2 E[e(n) x(n-i)] \\
& =-2 R_{e x}(i)
\end{aligned}
$$

From Eq.5, we can know that in order to make the MSE is zero, the best equalizer coefficient should be chosen such that the cross-correlation function of the input signal and the error function is zero. This means that the MSE can be taken to a minimum, when the input signal is orthogonal to the error function.

We can also see that the traditional serial equalization algorithm needs to provide the desired signal, which leads to a waste of channel bandwidth.

\section{Parallel Equalization Algorithm}

The Fig. 2 shows the parallel blind equalization algorithm structure in time domain. It differs from serial equalizer in that each module's variables are in vector form. And we adopt the improved parallel CMA in the error estimation module, which can avoid the LMS algorithm and the classic CMA defects.

We define the vector form of the input signal and the equalizer coefficient as follows

$$
\left\{\begin{array}{c}
X(n)=[x(n), x(n-1), x(n-2), \ldots, x(n-N+1)]^{T} \\
W(n)=\left[w_{0}(n), w_{1}(n), w_{2}(n), \ldots, w_{N-1}(n)\right]^{T}
\end{array}\right.
$$

The vector form of the Eq. 2 is written as

$$
W(n+1)=W(n)+\mu e(n) X^{*}(n)
$$


The single output of the equalizer is

$$
y(n)=W^{T}(n) X(n)
$$

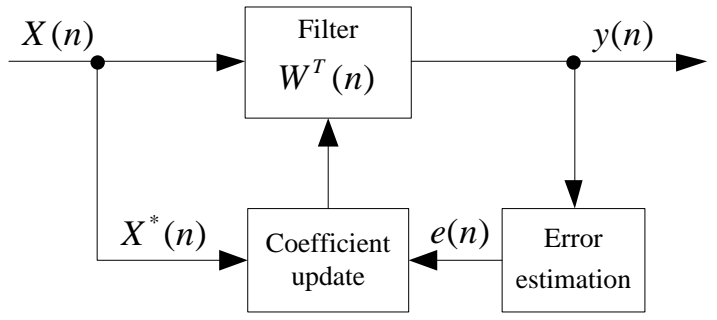

Figure 2. Parallel blind equalization algorithm structure

Suppose there is $L$ parallel input data, then Eq.7 can be expressed as

$$
\begin{aligned}
y(L k+i) & =W^{T}(L k+i) \cdot X(L k+i) \\
& \cong W^{T}(L k) \cdot X(L k+i) \quad(i=0,1, \ldots, L-1)
\end{aligned}
$$

where $k$ is the index value corresponding to $n$ in $L$ parallel.

Eq.6 is simplified as follows, which is based on summation approximation technique in relaxation advance transformation [10]. The simulation shows that this simplification does not significantly reduce the performance of the algorithm [11].

$$
\begin{aligned}
W[L(k+1)] & =W(L k+L-1)+\mu e(L k+L-1) X^{*}(L k+L-1) \\
& =W(L k)+\mu \sum_{m=0}^{L-1} e(L k+m) X^{*}(L k+m) \\
& \cong W(L k)+\mu \frac{L}{L A} \sum_{m=0}^{L A-1} e(L k+m) X^{*}(L k+m)
\end{aligned}
$$

We take $N=16, L=8, L A=2$ as an example, and Eq.8 and Eq.9 are as follows, respectively.

$$
\left\{\begin{array}{c}
y(8 k+i) \cong W^{T}(8 k) X(8 k+i) \quad(i=0,1, \ldots, 7) \\
W[8(k+1)] \cong W(8 k)+4 \mu \sum_{m=0}^{1} e(8 k+m) X^{*}(8 k+m)
\end{array}\right.
$$

The Eq.10 is expressed in the form of a matrix as follows

$$
\left[\begin{array}{c}
y(8 k) \\
y(8 k+1) \\
y(8 k+2) \\
\vdots \\
y(8 k+7)
\end{array}\right]=\left[\begin{array}{cccc}
x(8 k) & x(8 k-1) & \cdots & x(8 k-15) \\
x(8 k+1) & x(8 k) & \cdots & x(8 k-14) \\
x(8 k+2) & x(8 k+1) & \cdots & x(8 k-13) \\
\vdots & \vdots & \ddots & \vdots \\
x(8 k+7) & x(8 k+6) & \cdots & x(8 k-8)
\end{array}\right]\left[\begin{array}{c}
w_{0}(8 k) \\
w_{1}(8 k) \\
w_{2}(8 k) \\
\vdots \\
w_{15}(8 k)
\end{array}\right]
$$




$$
\left[\begin{array}{c}
w_{0}[8(k+1) \\
w_{1}[8(k+1) \\
w_{2}[8(k+1) \\
\vdots \\
w_{15}[8(k+1)]
\end{array}\right]=\left[\begin{array}{c}
w_{0}(8 k) \\
w_{1}(8 k) \\
w_{2}(8 k) \\
\vdots \\
w_{15}(8 k)
\end{array}\right]+4 \mu\left[\begin{array}{cc}
x^{*}(8 k) & x^{*}(8 k+1) \\
x^{*}(8 k-1) & x^{*}(8 k) \\
x^{*}(8 k-2) & x^{*}(8 k-1) \\
\vdots & \vdots \\
x^{*}(8 k-15) & x^{*}(8 k-14)
\end{array}\right]\left[\begin{array}{c}
e(8 k) \\
e(8 k+1)
\end{array}\right]
$$

According to Eq.11, Fig. 3 shows the filter structure of 8 parallel blind equalization algorithm. According to the equation (12), the filter coefficient update structure of 8 parallel blind equalization algorithm as shown in Fig.4.

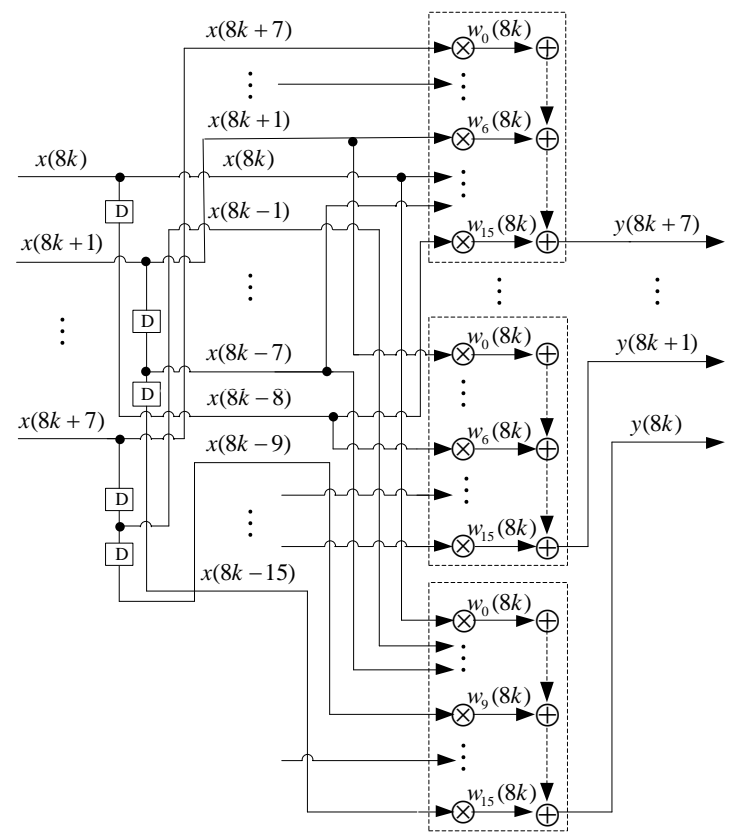

Figure 3.8 parallel filter structure

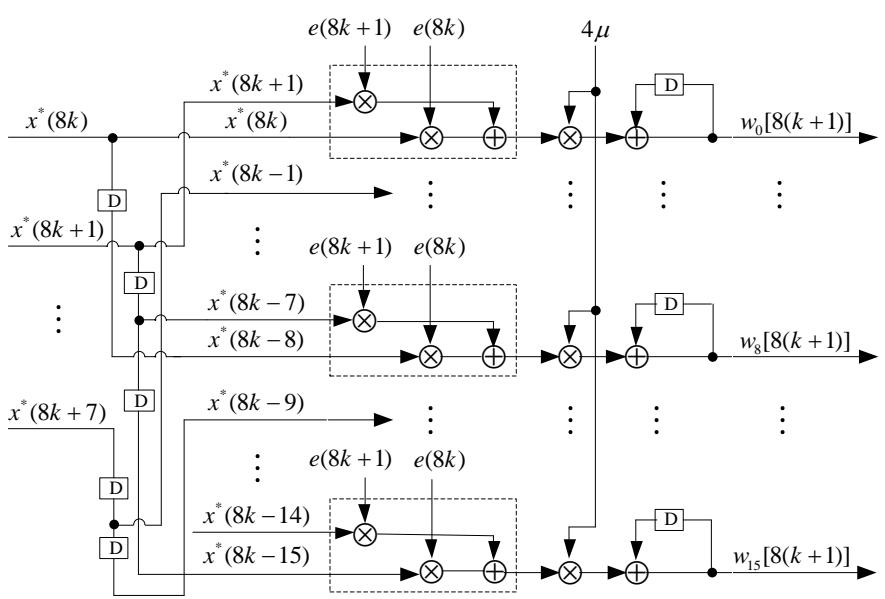

Figure 4. 8 parallel coefficient update structure 


\section{Classic CMA}

For the classic CMA algorithm, the error function $e(n)$ expression is

$$
\left\{\begin{array}{c}
e(n)=R^{2}-|y(n)|^{2} \\
R^{2}=E\left[|a(n)|^{4}\right] / E\left[|a(n)|^{2}\right]
\end{array}\right.
$$

where $R^{2}$ is the modulo value of the transmitted signal and is a fixed constant. The $y(n)$ represents the output signal of the equalizer. The $a(n)=a_{r}(n)+j a_{i}(n)$ is the information symbol at the transmitting side.

When the multi-mode signals is equalized with the classic CMA, the error value is not zero after equalization, because of the $R^{2}$ is a fixed modulus value. So there is a big misjudgment that is easy to produce a large MSE, and ultimately affect the equalizer effect.

\section{The Proposed CMA}

For multi-mode signals in the equalization process, we introduce the idea of coordinate transformation, and its principle shown in Fig.5.

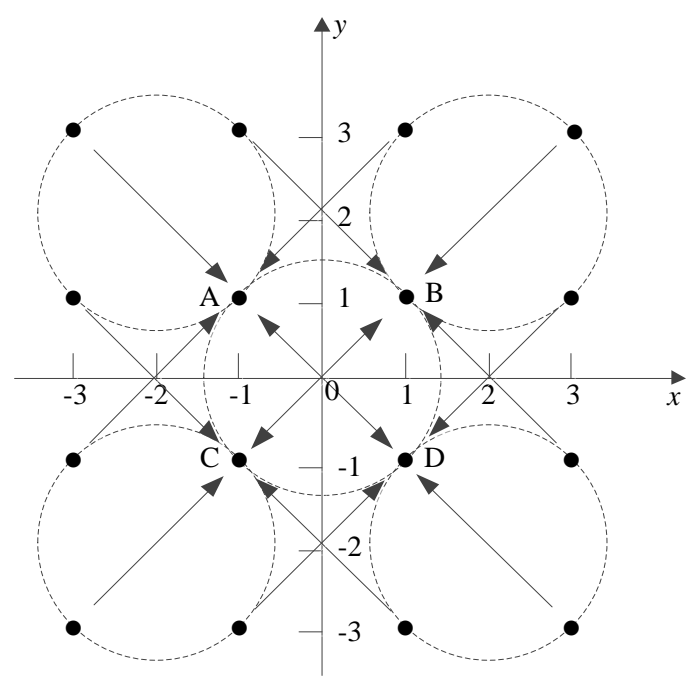

Figure 5. 16QAM coordinate transformation

In Fig.5, “•" represents an ideal 16QAM constellation point after equalization, which is distributed in four circles. The A, B, C, D are four points after coordinate transformation, and they are distributed on the same circle. Therefore, 16QAM signal constellation points are all transferred to A, B, C, D four points through coordinate transformation. When the signal is equalized, the difference between the transformed signal modulo value and the equalized signal is zero. Namely the error is 0 , the equalizer can have the best results.

After the coordinate transformation, the error function $e_{T}(n)$ is defined as follows

$$
e_{T}(n)=e_{T r}(n)+j e_{T i}(n)
$$

where $e_{T r}(n)$ and the $e_{T i}(n)$ are the real and imaginary parts of the error function, respectively. Its specific calculation expression is as follows 


$$
\begin{aligned}
& \left\{\begin{array}{l}
e_{T r}(n)=R_{T r}^{2}-\left|y_{T r}(n)\right|^{2} \\
e_{T i}(n)=R_{T i}^{2}-\left|y_{T i}(n)\right|^{2}
\end{array}\right. \\
& \left\{\begin{array}{l}
R_{T r}^{2}=\frac{E\left[\left|a_{r}(n)-2 \operatorname{sgn}\left[a_{r}(n)\right]\right|^{4}\right]}{E\left[\left|a_{r}(n)-2 \operatorname{sgn}\left[a_{r}(n)\right]\right|^{2}\right]} \\
R_{T i}^{2}=\frac{E\left[\left|a_{i}(n)-2 \operatorname{sgn}\left[a_{i}(n)\right]\right|^{4}\right]}{E\left[\left|a_{i}(n)-2 \operatorname{sgn}\left[a_{i}(n)\right]\right|^{2}\right]}
\end{array}\right. \\
& \left\{\begin{array}{l}
y_{T r}(n)=y_{r}(n)-2 \operatorname{sgn}\left[y_{r}(n)\right] \\
y_{T i}(n)=y_{i}(n)-2 \operatorname{sgn}\left[y_{i}(n)\right]
\end{array}\right.
\end{aligned}
$$

where $y_{r}(n)$ and the $y_{i}(n)$ represent the real and imaginary parts of the $y(n)$, respectively. The $\operatorname{sgn}[\cdot]$ represents a sign function, and its calculation process is as follows

$$
\operatorname{sgn}[x]=\left\{\begin{array}{cc}
1 & x>0 \\
0 & x=0 \\
-1 & x<0
\end{array}\right.
$$

According to Eq.12, we need two parallel error estimation structures. Combined with the proposed CMA, two parallel error estimation structure is shown in Fig.6.

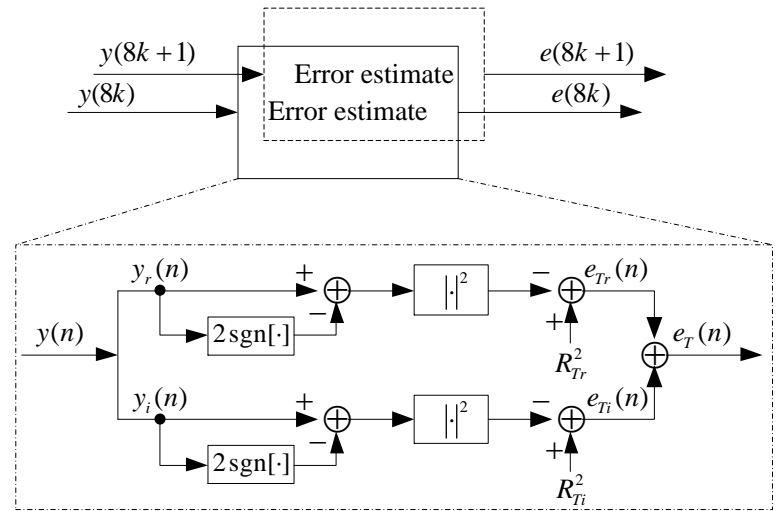

Figure 6. Two parallel error estimation structures

\section{Simulation Results}

In this section, the performance of the proposed entire high speed parallel blind equalization algorithm is shown, which is simulated in MATLAB. The simulation process is as follows. A 16QAM modulation signal is generated randomly at the transmitting module. The modulated 16QAM signal is through an Additive White Gaussian (AWG) noise channel, and the signal to noise ratio (SNR) is set to $25 \mathrm{~dB}$. Then, use the high speed parallel blind equalization algorithms in this paper at the receiving side. Fig.7 (a) shows the signal constellation of the receiving side. Fig.7 (b) is a constellation after equalization, which makes use of the classic CMA. After the proposed algorithm, the constellation is shown in Fig.7 (c). From Fig.7 (b), (c) and (d), we can see that the proposed parallel blind equalization algorithm works more 
efficiently compared to the classic CMA. Further, we use the (MSE) to measure the performance of high speed parallel blind equalization algorithms. Fig.7 (d) shows the MSE convergence curve. From the Fig.7 (d), it can be found that the error convergence speed of the proposed CMA is faster compared to the classic CMA, so that the equalizer output can be stabilized. Moreover, the MSE value of the proposed CMA is smaller than the classic CMA about $10 \mathrm{~dB}$.

Next, a communication channel filter is added to the simulation system, and the other simulation parameters are the same. This communication channel impulse response function is $\mathrm{h}=[0.3132,-0.104,0.8908,0.3134]$, and the magnitude $\&$ phase responses are given in Fig.8 (a). Due to the existence of the communication channel, the input signal constellation of the receiver is shown in Fig.8 (b). Fig.8 (c) shows the constellation after the equalizer, which uses the proposed parallel blind equalization algorithm. Fig.8 (d) is the MSE convergence curve. Compared with Fig.7 (d), it is found that the MSE value of the communication channel filter system is smaller, which indicates that the proposed parallel algorithm is quite efficient for non-ideal channel. It can be seen from Fig. 8 (b), (c) and (d) that the proposed parallel blind equalization algorithm is also very suitable for the communication system of complex environment.
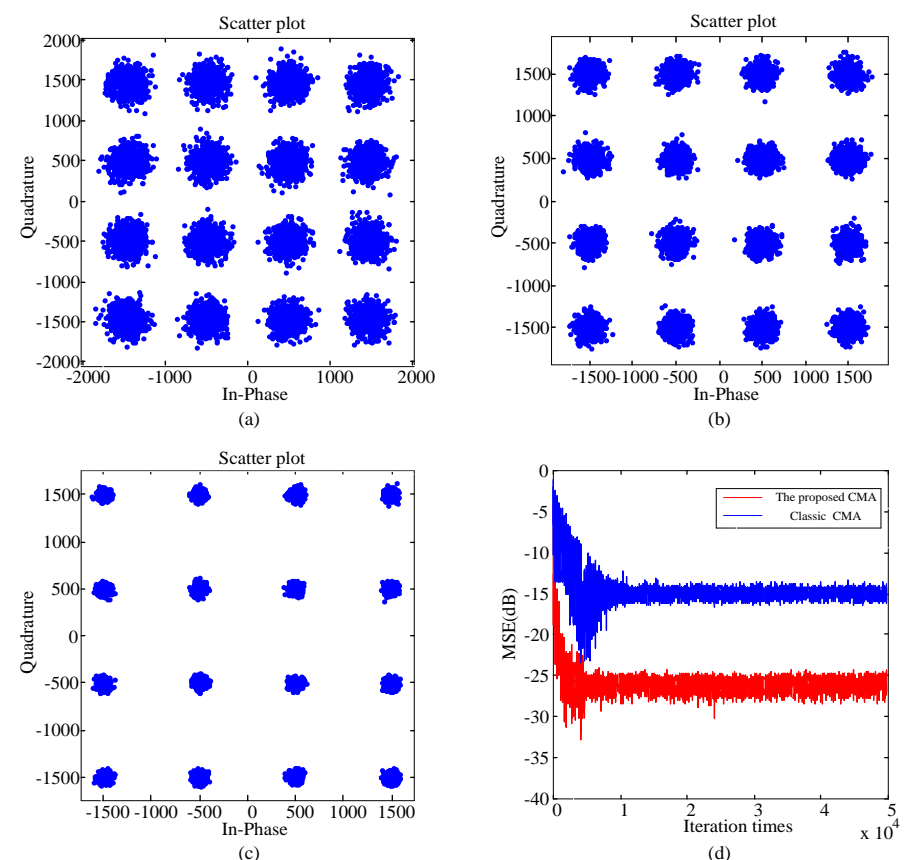

Figure 7. 16QAM simulation results 

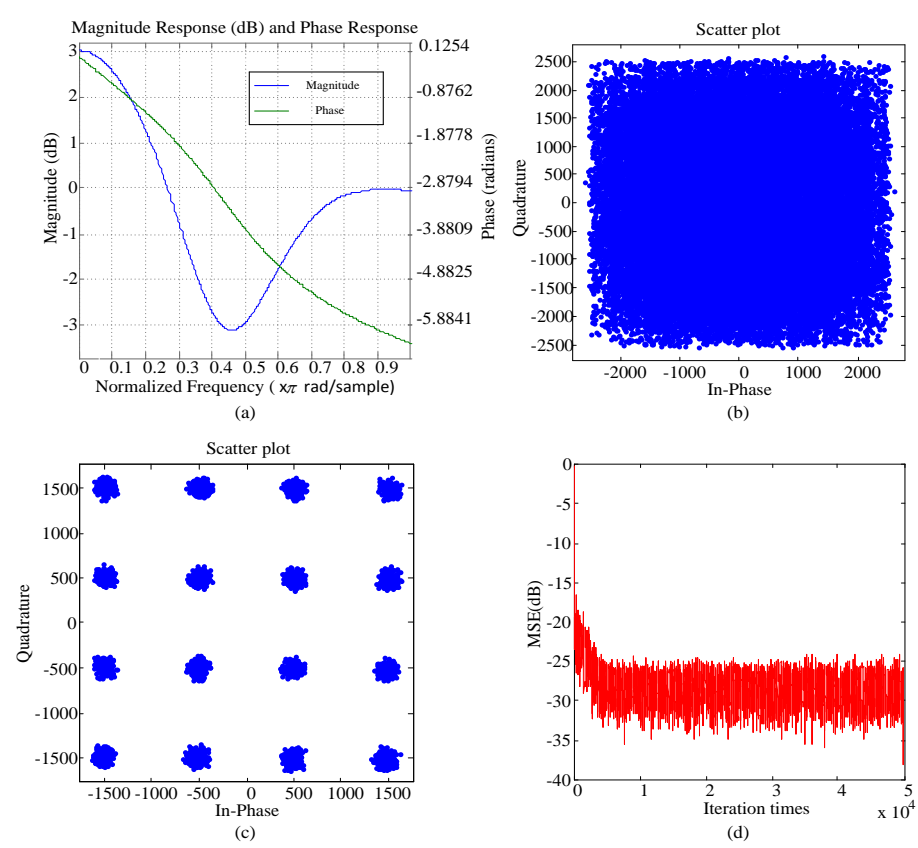

Figure 8. 16QAM simulation results with channel filter

\section{Conclusions}

In this paper a new fully parallel blind equalization algorithm is presented, which is very suitable for multi-mode signals. First, the traditional serial equalization algorithm has been studied. Then, we analyze the entire high speed parallel blind equalization algorithm structure, especially using the coordinate transformation formula in error estimation. The simulation results show that the performance of the new parallel algorithm is very excellent. This high speed parallel blind equalization algorithm can be widely used in wireless communication systems.

\section{Acknowledgement}

This research was financially supported by the National Natural Science Foundation of China (Grant No. 60934002, 61271035).

\section{References}

[1] Malik G, Sappal A S. Adaptive Equalization Algorithms: An Overview[J]. International Journal of Advanced Computer Science \& Applications, 2011, 2(3).

[2] Qureshi S U H. adaptive equalization[J]. IEEE Communications Magazine, 1982, 73(2):9-16.

[3] Sato Y. A Method of Self-Recovering Equalization for Multilevel Amplitude-Modulation Systems[J]. IEEE Transactions on Communications, 1975, 23(6):679-682.

[4] Godard D N. Self-Recovering Equalization and Carrier Tracking in Two-Dimensional Data Communication Systems[J]. IEEE Transactions on Communications, 1980, 28(11):1867-1875. 
[5] Treichler J, Agee B. A new approach to multipath correction of constant modulus signals[J]. IEEE Transactions on Acoustics Speech \& Signal Processing, 1983, 31(2):459-472.

[6] Treichler J R, Wolff V, Johnson C R. Observed misconvergence in the constant modulus adaptive algorithm[C]// Asilomar Conference on. IEEE, 1991:663-667 vol.2.

[7] Proakis J G. Digital Communications, 3rd Edition[J]. MA : McGraw-Hill, 2001.

[8] Jacobs I. Tracking issues of some blind equalization algorithms[J]. IEEE Signal Processing Letters, 2004, 11(9):760-763.

[9] Sayed, Ali H. Fundamentals of Adaptive Filtering[J]. Control Systems IEEE, 2003, 25(4):77 - 79.

[10]Parhi K K. VLSI Digital Signal Processing Systems: Design and Implementation[J]. Springer, 1999, 9(1):165 - 170.

[11] Shanbhag N R, Parhi K K. Relaxed Look-Ahead Pipelined Lms Adaptive Filters And Their Application To Adpcm Coder[J]. Circuits \& Systems II Analog \& Digital Signal Processing IEEE Transactions on, 1993, 40(12):753 - 766. 